\title{
Famille et élevage : sens et organisation du travail
}

\author{
Cécile Fiorelli1', Jocelyne Porcher², Benoit Dedieu ${ }^{3}$
}

\section{Introduction}

Les relations entre famille et exploitation agricole sont aujourd'hui l'objet de tensions : d'un côté, les agriculteurs sont enjoints d'être de vrais chefs d'entreprise (Rémy, 2011), ce qui fait abstraction des dimensions identitaire, patrimoniale et affective de ce métier ; d'un autre côté dans les discours sur et dans l'accompagnement à l'installation agricole, il n'a jamais été autant question d'articuler projet de vie et projet professionnel ${ }^{4}$. Par ailleurs, on entend souvent les éleveurs et leurs représentants déplorer leur surcharge de travail, leur manque de disponibilité pour leur famille (Couzy et Dockes, 2006), et, pour certains, espérer que leurs enfants fassent un autre travail, mieux rémunéré et leur laissant plus de disponibilités (Fillonneau, 2012). Ces tensions renvoient à ce que Barthez a appelé le choc entre les rapports de travail et des rapports familiaux sur l'exploitation agricole familiale (Barthez, 1996). Ils renvoient au " passage d'une agriculture vécue comme un état, une manière d'être, un mode de vie, à une agriculture comme activité professionnelle ; c'està-dire un secteur de vie dans un ensemble plus vaste " (ibid., p. 15). "Du travail comme mode de vie on est passé à un travail comme facteur de production ; c'est beaucoup plus restrictif, mais cela entrâne des exigences très précises. Il y a désormais deux entités : l'entreprise et la famille. Cette entreprise doit être rentable, doit fournir un revenu pour nourrir la famille " (ibid., p. 16). Ces différents éléments interrogent particulièrement la façon dont la famille est engagée dans le travail agricole sur les exploitations familiales ou dont le travail agricole engage la famille.

1. INRA, UMR 1273 Métafort, 63122 Saint-Genes-Champanelle, France, cecile.fiorelli@clermont.inra.fr

2. INRA, UMR 951 Innovation, 34070 Montpellier, France, jocelyne.porcher@supagro.inra.fr

3. INRA, UMR 1273 Métafort, 63122 Saint-Genes-Champanelle, France, benoit.dedieu@clermont.inra.fr

4. http://www.jeminstallepaysan.org

Ce chapitre de l'ouvrage L'agriculture en famille : travailler, réinventer, transmettre est publié en Open Access sous licence creative commons CC-BY-NC-ND permettant l'utilisation non commerciale, la distribution, la reproduction du texte, sur n'importe quel support, à condition de citer la source. 
Des études en sociologie rurale soulignent la diversité actuelle des rapports au travail, rapport au temps de travail, qui ne se limitent pas à un rapport strictement économique (Dufour et Dedieu, 2010 ; Fillonneau, 2012). Ces études analysent plutôt ces rapports au travail en ce qu'ils placent l'individu ou non en difficulté avec des normes socio-professionnelles. Par ailleurs, les études portant sur l'organisation du travail sur les exploitations d'élevage familiales, principalement portées par les zootechniciens, ont mis en évidence la diversité des types de tâches et des quantités de travail réalisées par les membres de la famille (Madelrieux et al., 2009; Hostiou et Dedieu, 2012) ainsi que la diversité des configurations familiales engagées dans ce travail d'élevage (agriculteur seul, couple, associés familiaux, avec l'aide de parents...). Mais le sens donné au travail, ce qui fonde l'engagement de l'individu au travail n'est pas abordé dans ces deux ensembles d'études. De même, si le point de vue du chef d'exploitation ou bien spécifiquement celui des femmes (Dufour et al., 2010), est pris en compte, ce que traduit l'ensemble des membres de la famille travaillant sur l'exploitation demeure inconnu. Or tous les membres n'ont pas la même relation à l'exploitation (Bessière, 2008), ils n'envisagent pas de la même façon le travail agricole et sa place dans la vie de la famille (Terrier et al., 2012).

Notre contribution vise à éclairer la diversité du sens et des modalités d'engagement de la famille dans le travail agricole sur des exploitations d'élevage familiales françaises au début des années 2000. Pour cela, nous nous appuyons sur une étude conduite dans le cadre d'une recherche doctorale (Fiorelli, 2010) auprès d'éleveurs pluriactifs, c'est-à-dire de personnes qui font de l'élevage et qui exercent au moins une autre activité professionnelle à titre salarié ou indépendant. Ces situations particulières ont été retenues car elles exacerbent les difficultés de conciliation de l'élevage et de la famille, du fait des contraintes d'organisation du travail et de la quantité de travail effectuée (Blanchemanche, 2000). Nous présenterons d'abord le cadre théorique auquel se réfere l'étude, ainsi que quelques éléments de méthodologie. Puis nous illustrerons plusieurs cas relevant de situations d'individus (le conjoint étant peu présent sur la ferme) ou de couples au travail.

\section{Cadre théorique}

Pour décrire et appréhender la manière dont les éleveurs s'engagent différemment dans le travail, nous mobilisons les concepts de la psychodynamique du travail (Dejours, 2009). Cette discipline est fondée sur une approche psychanalytique de l'individu au travail, éprouvant des désirs inconscients et contradictoires, éprouvant du plaisir et de la souffrance, investissant sa subjectivité dans le travail. Elle s'intéresse au pouvoir du travail "de faire advenir le sujet» (Dejours, 1998, p. 7). En effet, elle considère que " travailler, c'est investir sa subjectivité ", " travailler, c'est mobiliser son corps, son intelligence, sa personne, pour une production ayant valeur d'usage ". Pour Dejours (2009), "le travail est toujours une mise à l'épreuve de la subjectivité, dont cette dernière sort accrue, grandie ou au contraire, rétrécie, meurtrie. [...] Travailler, c'est non seulement produire, c'est aussi se transformer soi-même et, dans le 
meilleur des cas, c'est une occasion offerte à la subjectivité de s'éprouver elle-même, voire de s'accomplir $"(p .15)$.

Le rapport subjectif au travail est la relation que le sujet entretient avec son travail, c'est-à-dire ce qu'il engage de lui dans le travail et ce qu'il en attend. Cette notion est proche des notions de conceptions de métier et de motivations mais elle s'en distingue. En effet, la notion de conception de métier utilisée en sociologie réfere à l'inscription dans un champ professionnel et à la dimension identitaire du travail (Lémery, 2005 ; Dufour et Dedieu, 2010). Elle n'intègre pas les questions de l'affectivité dans les relations de travail, ni d'engagement du corps dans le travail. La notion de motivation renvoie à des cadres théoriques de psychologie comportementale, dans lesquels l'inconscient et la subjectivité sont absents. Ainsi le rapport subjectif au travail est complexe, multidimensionnel et personnel. En élevage, Porcher (2002) a montré l'importance de la rationalité relationnelle et en particulier affective du travail avec les animaux ; Mouret (2012) s'est intéressé à la rationalité morale.

Dans l'étude présentée ici, la place de la famille dans le sens donné au travail est caractérisée à l'aide de la grille d'analyse du rapport subjectif développée pour analyser la façon dont les éleveurs parviennent ou non à concevoir leurs systèmes d'élevage et à organiser leur travail de manière satisfaisante au regard de leur rapport au travail (Fiorelli et al., 2010). La grille est constituée des rationalités suivantes : économique (attentes de revenu), technique (intérêt pour la maîtrise technique et organisationnelle du processus et des performances de production), relationnelle (relations entre travailleurs et avec les animaux), identitaire (développement personnel et inscription dans un champ professionnel), relative à l'engagement du corps dans le travail (nature de la mobilisation du corps dans le travail).

L'organisation du travail est abordée par les zootechniciens comme l'agencement à différentes échelles de temps (journée, semaine, saison, année) des tâches découlant des choix de conduite d'élevage et des cultures (quotidiennes et non quotidiennes, avec les animaux ou sur les surfaces) et de l'ensemble des membres du collectif de travail, c'est-à-dire l'ensemble des personnes qui travaillent sur l'exploitation à un moment ou à un autre de l'année, quel que soit leur statut (Madelrieux et Dedieu, 2008 ; Hostiou et Dedieu, 2012). Nous nous centrerons dans ce texte sur la caractérisation de la place de la famille dans le collectif de travail et dans la répartition des tâches.

\section{Matériel et Méthode}

Nous avons réalisé une étude auprès de sept ménages conduisant des exploitations ovines dans le Puy de Dôme (France), choisis pour la diversité de leurs contraintes d'organisation du travail, et de leurs attentes vis-à-vis de l'élevage identifiées au cours d'une précédente étude (Fiorelli et al., 2007). Quatre enquêtes successives entre 2004 et 2007 ont permis d'aborder : l'approche globale des activités professionnelles 
et sociales du ménage et plus particulièrement de l'activité agricole, les représentations de l'élevage de chaque adulte du ménage impliqué dans l'activité d'élevage, la conduite de l'élevage et des cultures, l'organisation du travail. Le rapport subjectif au travail a été caractérisé à partir de l'analyse de discours obtenu au cours de l'entretien compréhensif portant sur les représentations de l'élevage (Sens et Soriano, 2001), analyse mise ensuite en débat auprès de chaque éleveur, puis complétée et illustrée à partir de l'analyse des pratiques et des modalités d'organisation.

Tous les adultes du ménage travaillant régulièrement sur l'exploitation ont été enquêtés. Six d'entre eux travaillaient en couple sur l'exploitation et exerçaient chacun au moins une activité professionnelle à l'extérieur de l'exploitation. Quatre travaillaient seuls sur l'exploitation et exerçaient au moins une autre activité professionnelle à l'extérieur de l'exploitation.

Pour analyser les formes d'engagement de la famille dans le travail agricole, nous avons caractérisé trois éléments : (i) la façon dont la famille est convoquée dans le rapport subjectif au travail ; (ii) la part familiale et le rôle des membres de la famille dans le collectif de travail et la façon dont les rationalités de chacun interagissent ; (iii) la place de la famille dans la gestion du temps en précisant les relations entre temps dédié à l'élevage, aux autres activités professionnelles et à la famille.

\section{Résultats}

Nous présentons trois cas emblématiques de la diversité des engagements dans le travail agricole de la famille : de l'engagement familial le plus modéré à l'engagement familial le plus fort, à la fois dans le sens donné au travail et dans la façon de travailler.

\section{Georges}

\section{L'élevage, une activité de réalisation personnelle}

Georges a 53 ans. Il s'est installé en 1994 lors du départ à la retraite de son beau-père après avoir travaillé 15 ans avec lui. Il élève 265 brebis. Depuis 25 ans, il travaille deux nuits toutes les quatre nuits dans un centre de tri. Il n'a pas choisi ce travail " alimentaire ", "répétitif ", sans marge de manœuvre pour décider ou s'organiser, mais qui "lui laisse pas mal de temps». L'élevage pour lui, c'est : "un loisir, ce n'est pas une contrainte, c'est... C'est un plus dans ma vie quoi"; " [...] je ne trouve pas le mot. Ce n'est pas un passe-temps, ce n'est pas un loisir parce que quand même c'est du boulot, mais c'est, j'ai l'impression de faire quelque chose "; "c'est quelque part progresser ". Georges oppose l'élevage et son travail à la Poste : «[...] mon activité à la Poste ne mapporte pas vraiment de, [...], enfin peu de satisfaction, je ne m'en cache pas, il n'y a pas de sot métier, mais je n'ai pas... Quand j'ai commencé, jai dit, si je dois faire ça toute ma vie, je n'ai pas... Je n'y voyais pas beau comme on dit. ". Au contraire, l'élevage 
est une activité de réalisation personnelle, qui donne la possibilité de progresser et d'entreprendre : «[...], par rapport à la Poste, que je fasse bien mon boulot ou mal, j’ai la paie à la fin du mois quoi, là si je le fais bien ou mal il y a une différence ". "Et puis aussi bien sûr [...], je peux prendre des décisions, à moi, et qui influencent direct sur ce qu'il y a, sur après quoi, ou sur mon temps libre et ainsi de suite quoi. Alors que par rapport à mon activité principale là je n'ai pas de choix".

Georges est fier d'être reconnu comme un paysan, avoir appris à élever des bêtes, d'obtenir de bons résultats techniques (il regarde plus le nombre d'agneaux que la marge), de gérer l'exploitation à sa manière, et de parvenir à ne pas être l'esclave de son travail. La dimension patrimoniale n'est pas présente dans le discours de Georges, il loue l'essentiel des terrains et ses deux filles ne souhaitent pas reprendre l'exploitation.

\section{Un collectif de travail important, relativement indépendant de la famille, gage de liberté}

Le collectif de travail rassemble une dizaine de personnes dont l'implication se limite au travail non-quotidien. Au quotidien, Georges met un point d'honneur à assurer seul le travail avec les brebis, car il aime décider et s'organiser comme il l'entend : "Maintenant comme je suis tout seul, je fais carrément ce que je veux!", "je vais à la Poste, ma femme ne met pas les pieds, mais c'est aussi tacite parce que je ne veux pas qu'elle vienne pour d'autres raisons. Ma femme elle bosse aussi ».

Pour se faciliter le travail avec les animaux, Georges a récemment appris à travailler avec un chien : "Sans chien je peux y amener à la foire", "comme dit le moniteur, déplacer les brebis ça doit être un plaisir, pas un stress", "je n'en suis pas encore au plaisir, mais jai moins de stress".

En outre, pendant les agnelages d'hiver, Georges se fait parfois remplacer à La Poste pour être moins fatigué. Seule une autre personne de la famille de Georges contribue régulièrement au travail agricole : son beau-père qui a plus de 80 ans. Sa contribution diminue du fait de son âge et elle est de moins en moins appréciée par Georges. Son rôle, aujourd'hui, consiste aussi à donner l'alerte s'il y a un problème avec les bêtes quand Georges dort en journée ou quand il est parti en vacances. La femme de Georges intervient trois fois par an : au moment de la tonte, pour relever les numéros et pour deux déplacements de troupeau.

Les autres membres du collectif n'appartiennent pas à la famille (un voisin agriculteur, deux entreprises de travaux agricoles, un salarié de Cuma) et assurent la quasi-totalité du travail non quotidien sur les surfaces (hormis la fauche) ainsi que le curage du bâtiment. Georges préfere payer ces travaux pour réduire le stress pendant les foins, limiter ses besoins en matériel, se libérer du temps pour ses loisirs la semaine et le week-end et ses vacances : il utilise ses congés de la Poste pour partir en vacances et non pour les travaux agricoles. Il ajoute qu'il considère que l'organisation est plus simple et que le travail est mieux fait. Pour lui, la sous-traitance du travail à son voisin est aussi une manière d'entretenir de bonnes relations avec ses pairs, dont la reconnaissance est très importante pour lui. 
Pour le remplacer pendant ses vacances, il a expérimenté plusieurs modalités d'organisation. D'après lui, "le seul souci possible par rapport aux bêtes, c'est l'attaque de chiens » et le travail à faire est simple, il suffit d'enlever une clôture pour agrandir la zone à pâturer. "Jusqu'à présent, il y avait un gars dans le village, c'était un jeune, il connaissait un peu l'élevage, payé au noir, ça avait toujours très bien marché" . Ce jeune est parti, et c'est le beau-père qui a fait l'intérim pendant que Georges était quatre semaines en vacances. Il vient de se renseigner pour faire appel au service cantonal de remplacement. Il trouve le prix acceptable mais il n'est pas sûr que cela lui convienne du fait que le remplaçant n'est pas sur place.

\section{Articulation des temps de travail agricole, non agricole et des temps de non-travail}

Pour Georges, à l'exception des quatre semaines d'agnelage d'hiver, le temps dédié au travail agricole est subordonné au quotidien à son travail de salarié mais aussi à ses loisirs et ses vacances. Ainsi Georges fait partie des pluriactifs qui distinguent temps pour l'élevage, temps pour le travail salarié à la Poste et temps de non-travail, en particulier du temps pour son couple, ses filles adultes qui habitent loin, pour des activités de loisir (musique, danse, voyages). De ce fait, il a envie de ne consacrer à l'élevage qu'une partie de son temps laissé disponible par son travail salarié. Il aime aller danser chaque semaine, quand il ne travaille pas la nuit : "Ça veut dire que pour le boulot, à la bergerie, j’en fais plus le matin et quand on a dansé le dimanche aprèsmidi, le soir je ne me couche pas bien de bonne heure".

Le travail à la Poste contraint peu les choix de conduite du troupeau (période de reproduction, gestion de l'alimentation, du renouvellement, de la conduite sanitaire, etc.) ; la conduite est plutôt influencée par les vacances qui conditionnent les débuts et fin d'agnelage, pour qu'il y ait le moins possible d'agneaux à nourrir pendant son absence.

L'engagement de Georges dans le travail agricole relève surtout de la réalisation personnelle et moins d'un revenu complémentaire, à côté d'un travail salarié jugé inintéressant mais rapportant un revenu confortable. Même si l'exploitation est d'origine familiale, la dimension familiale de l'engagement est très restreinte : Georges met en avant la possibilité de développement personnel ; le collectif est essentiellement non familial en dehors de Georges et de son beau-père ; temps de travail agricole et temps en famille sont distincts.

\section{Robert et Béatrice}

\section{L'élevage, une façon de faire famille}

Robert et Béatrice, élèvent 340 brebis sur 99 ha. Béatrice est salariée dans un supermarché où elle fait de la mise en rayon six jours par semaine de 5 heures à 10 heures. Elle a 38 ans et n'a pas d'enfant. Robert a 56 ans et sa fille n'habite plus avec eux. 
Il a développé une activité saisonnière d'entretien de voirie auprès des communes alentours.

Béatrice raconte qu'elle n'a "pas d'enfants à deux pattes mais elle en a beaucoup à quatre pattes ", avec Robert, avec qui elle partage la même passion, donc "ça va tout seul pour travailler ensemble ". Son engagement dans le travail agricole est marqué par un très fort investissement affectif dans les relations avec les brebis et les agneaux. Elle insiste sur le fait qu'elle est devenue "une gaga des moutons ", qu'elle " aime trop les bêtes", "leurs filles». C'est un plaisir partagé avec Robert mais avec des nuances : "[...] il a toujours aimé ses bêtes mais peut-être pas au point de... de les voir comme des gamins quoi. Enfin, c'est bête à dire mais...Moi, j’y vais, c'est mes bébés quoi. Donc, quand je vais à la bergerie, c'est mes filles, [...] Moi, mes chiens, c'est pareil, c'est mes bébés. Si je leur dis : allez chercher votre papa, je ne leur dis pas le patron, je leur dis "le papa", ils vont chercher Robert. On n'a pas d'enfant nous et... [...] L'amour comme je n'ai pas d'enfants, je le remets sur les bêtes [...]. Moi, je n'ai pas de petiots alors... [...] Enfin, moi on m'a dit que je les aimais trop. C'est vrai mais bon". Faire de l'élevage c'est une façon d'être maman pour Béatrice, et faire de l'élevage avec Robert, c'est une façon de faire famille.

La pratique d'élevage la plus emblématique de cette façon de voir l'élevage est le choix de donner des biberons aux agneaux, abandonnés ou dont les mères n'ont pas assez de lait, malgré la pénibilité de cette tâche, le temps qu'elle requiert ainsi que son coût. Ainsi Béatrice parle d'elle comme la "Mère Biberons ", irremplaçable et de Robert comme le "Père Fouettard": "Parce que Robert quand on lâche, on trie les agneaux, il trie au fouet. Donc moi je suis la Mère Biberons et lui quand ils le voient c'est le Père Fouettard, donc même sil a les biberons à la main, ils ne vont pas venir. [...] Même avec une gastro, une grippe, je suis obligée de descendre faire boire les agneaux, ils ne boivent pas avec lui. "Robert justifie cette pratique par le respect de la vie et l'amélioration des résultats techniques : ça évite de tuer des agneaux, il dit qu'il n'en a pas le courage, et ça permet de réduire le taux de mortalité. Il ne parle pas du coût du lait en poudre dont les frais sont les plus élevés parmi l'ensemble des éleveurs ovins suivis par Laignel et al. (2008).

Tous les agneaux sont soignés, alimentés, élevés quels que soient le temps, l'énergie et l'argent qu'il faudra. Pour Robert, "les bêtes rendent ce que vous leur faites " et donc il a tout intérêt à bien s'occuper des bêtes, même si cela coûte cher : "Voilà, au lieu d'avoir des bêtes plus malades ou des bêtes... moi si je les entretiens... et puis elles font de jolis agneaux. Toute manière plus vous les soignez euh... plus vous leur donnez et plus elles vous font des agneaux ». Pour Béatrice, c'est une question d'attachement à chacune des bêtes : elle baptise notamment les agnelles élevées au biberon. Elle aime qu'elles la reconnaissent, qu'elles viennent quand elle les appelle et la suivent ensuite " comme des chiens». Elle se souvient de leur date anniversaire, elle s'arrange avec Robert pour ne pas les vendre et les garder pour le renouvellement du troupeau habituellement acheté à l'extérieur.

Si Béatrice et Robert partagent un très fort investissement affectif avec les bêtes, leur rapport à l'élevage differe toutefois par la place des rationalités économiques et techniques : Béatrice les nie, alors qu'elles ont leur place dans les choix de Robert 
pour qui l'élevage constitue l'essentiel de son revenu : "C'est-à-dire au départ j'ai choisi ça parce que mes parents avaient toujours des moutons puis, voilà. Et puis c'est vrai que ça gagne un peu sa vie... " Béatrice raconte que c'est plutôt Robert qui décide de continuer à soigner une brebis ou la faire euthanasier car sinon elle engagerait des dépenses trop importantes, parce qu'il a un "roulement à faire ", "c'est son gagnepain " contrairement à elle, qui déclare que les résultats économiques de l'élevage n'ont pas d'importance pour elle, "Non, je les aime trop pour en faire un métier ". Elle précise qu'elle a "[sa] situation, [son] compte bancaire, [sa] voiture " et qu'ils ont " toujours été là-dessus indépendants".

\section{Collectif de travail et répartition des tâches}

Le collectif de travail est essentiellement composé de deux personnes, Béatrice et Robert qui travaillent tous les jours sur l'exploitation et assument l'ensemble du travail quotidien avec le troupeau. Ils ne mobilisent des aides que très ponctuellement pour la tonte et la moisson.

Chez Béatrice et Robert, la répartition des tâches renvoie à deux logiques : le plaisir pour Béatrice et Robert de travailler ensemble avec les animaux, la peur des tracteurs de Béatrice. Travailler ensemble est plus important que de gagner du temps, c'est un élément fort dans l'organisation. En résumé, Béatrice et Robert assurent ensemble le travail avec le troupeau, Robert s'occupe seul des travaux sur les surfaces (prairies, parcours, cultures), Béatrice est la seule à pouvoir distribuer des biberons.

\section{Gestion du temps}

Le travail avec les animaux est subordonné à l'activité de salariée au supermarché de Béatrice : il commence à son retour vers 10h30. L'activité d'entretien de voirie de Robert est subordonnée à l'activité agricole (élevage et cultures), il n'a pas de pression sur les dates de finition des chantiers.

Le temps de l'élevage est souvent un temps avec Robert, et avec les "filles ", les brebis, d'une certaine façon le temps de l'élevage est perçu comme du temps en famille, et pas toujours comme du temps de travail. Ainsi Béatrice raconte qu'elle ne voit pas le temps passer quand elle va "parler" aux brebis avec Robert ou qu'elle a plaisir à passer du temps "au milieu " des brebis: "Et l'été quand elles sont dehors, je vais au pré le soir vers 7 heures quand il fait frais et puis je m'assois au milieu et puis je reste deux heures comme ça. Et puis là je suis bien ». Tout se passe comme si le temps de l'élevage sans cadence ni horaires imposés reposait du temps de l'autre travail beaucoup plus dense : "C'est comme quelqu'un qui va au sauna, prendre une heure de sauna, moi je vais dans la bergerie et par rapport, au boulot, qu'on est à 100 à l'heure pendant cinq heures, que c'est stressant, qu'on se fait engueuler, machin, tu te dis, je suis bien là ".

Il n'y a quasiment pas d'autres temps que le temps dédié au travail agricole, au supermarché ou à l'entreprise. Béatrice raconte : "Moi le soir à 9 heures, il n'y a plus de bonne fermme. On ne peut rien faire. On ne peut pas dire, samedi on va boire l'apéro chez quelqu'un. Non je vais me coucher. On n'a pas de vie. Mais c'est un choix pour 
ça, pour les bêtes". Béatrice et Robert ne partent pas en vacances. Béatrice pose des congés pour l'agnelage d'octobre, la tonte en avril et l'enrubannage en mai-juin. Robert ne fait pas de chantier d'entretien de voirie pendant l'enrubannage en juin, ni pendant l'agnelage d'octobre. Béatrice et Robert limitent le travail agricole les dimanches : ils ne font que le travail quotidien non reportable (alimentation des brebis et des agneaux).

Béatrice et Robert ne comptent pas le temps passé avec les bêtes : "Demain si on descend soigner, mettons, à 11 heures et demie, on va remonter à 3 heures et demie, 4 heures. Parce que, le temps que moi je leur ai parlé, que lui, il leur ait parlé, qu'il fasse le tour avec la pougnette de grain [...], mais ça, ça ne vient pas de moi! [...] Mais en fait quand on est en bas on ne voit pas le temps passer». En revanche, pour s'affranchir de ce qu'ils n'aiment ni l'un, ni l'autre, les horaires imposés fixes, et pour réduire le temps de travail quotidien en hiver, Béatrice et Robert ont organisé un système de libre-service pour alimenter les brebis et les agneaux (des balles rondes disposées au milieu de la bergerie pour les brebis et un tuyau branché sur un silo à grain pour les agneaux). Mis à part les agneaux aux biberons, grâce à l'équipement et à un compromis sur les coûts d'alimentation, ils peuvent se permettre de distribuer l'alimentation tous les deux ou trois jours : "[...] pour ce qui est de soigner, de nettoyage, enfin tout ce qui n'est pas..., il a vachement simplifié, on est tranquille, l'hiver, nous, en 1 1h30 on soigne 400 moutons pour trois jours. " Pour Robert, "ça simplifie un peu les choses, quoi; le matin on y va quand on veut, le soir on y va quand on veut, du moment qu'elles ont à bouffer".

L'engagement dans le travail agricole de Béatrice et Robert relève d'un engagement familial très fort. Certes Robert a hérité de la petite exploitation de ses parents, mais ce qui est le plus marquant c'est que l'élevage leur permet de faire famille et de vivre en famille. Linvestissement affectif dans les relations avec les animaux est très fort, maternel, de la part de Béatrice ; de la part de Robert, il relève davantage du respect de la vie. La quasi-totalité du travail agricole est réalisée par le couple. Le temps de travail agricole est perçu comme un temps ensemble en couple et en famille avec les bêtes. Ils ne cherchent pas à en réduire la durée mais à se "simplifier la vie " par des aménagements des bâtiments et des parcelles.

\section{Lucien et Marie-Françoise}

\section{Travailler en famille comme façon de vivre en famille}

Chez Lucien et Marie-Françoise, le principe est le suivant : toute la famille pour faire le travail et du travail pour toute la famille. Ils tiennent une boulangerie-pâtisserie ouverte six jours sur sept, toute l'année sauf en novembre et élèvent 200 brebis et une quinzaine de chevaux de trait en race pure pour les concours. Ils ont trois fils, l'aîné veut devenir éleveur, le cadet boulanger.

La pluriactivité est une affaire de famille depuis plusieurs générations : "Mon père, bon il faisait les vaches, mais j'avais ma grand-mère, parce que c'était tout en famille! 
[...] À part ma mère qui faisait la vente. Et la tante elle faisait le pain la nuit, après elle prenait son râteau, elle allait tourner le foin, et tout, et la grand-mère aussi! ". Lucien n'a pas choisi d'être boulanger, il a dû remplacer son père qui était malade, il aurait voulu être éleveur. Il a repris les chevaux et la boulangerie à la suite de son père et de sa tante. Aujourd'hui, il travaille avec sa femme et deux de ses fils. Travailler avec les bêtes c'est être dehors, à l'extérieur du fournil, "tranquilles ». Ils ne visent pas à obtenir un revenu. Lucien déplore que les moutons lui demandent autant de travail. Il n'aime pas les brebis mais il a constitué une exploitation d'élevage qu'il est content de transmettre à son fils : "J'en avais sept-huit, c'est tout. Et après quand ily a eu les fils qui ont voulu, ils veulent faire ça, et on s'est mis aux moutons. On a acheté des moutons. [...]. Il y en a un qui va passer son BEP agricole, il veut faire ça". MarieFrançoise explique que l'exploitation est déjà un peu celle de son fils : «[...] de toute façon ce sera pour lui donc... il fait comme si c'était à lui ». Les chevaux leur apportent beaucoup de fierté, dans les concours de race. Pour les chevaux, ils n'hésitent pas à investir beaucoup de temps et d'argent pour les frais vétérinaires.

En parlant de l'élevage, Marie-Françoise dit : "En fait je pense que, [l'élevage] c'est pour nous dépayser...se sortir un peu de là, penser à autre chose et voir autre chose [...] C'est le fait de carrément couper les ponts, quoi. Quand tu es avec les bêtes tu ne penses pas [...], quand tu es à la boulangerie faut toujours penser est-ce que je n'ai pas oublié ça, bien fait ça, est-ce que celui-là a été servi est-ce que...faut toujours être sur le quivive, [...] alors qu'avec les moutons, non ». Elle a une formation initiale agricole faite plutôt par défaut et souligne que l'activité ovine ne s'inscrit pas dans la continuité de cette formation. Pour elle, l'élevage, "c'est plus un loisir, [...], un plaisir ", " ça change du commerce ". "C'est un autre train de vie ". Marie-Françoise oppose de manière caricaturale son travail à la boulangerie et l'élevage, comme si l'activité d'élevage ne présentait aucune contrainte jusqu'à la fin de l'entretien où elle aborde l'absence de revenu issu de l'élevage et celui important de la boulangerie. Marie-Françoise ne voit donc pas l'élevage comme du travail supplémentaire mais comme une activité qui détend après le travail de la boulangerie.

\section{Un collectif de travail essentiellement familial, très polyvalent}

Lucien et Marie-Françoise rassemblent un collectif de travail essentiellement familial de onze personnes. Le nombre de travailleurs réguliers est conséquent, en cohérence avec les activités du ménage : fabrication, tournées, livraison, vente au magasin pour la boulangerie-pâtisserie, élevage équin avec préparation des animaux pour les concours, élevage ovin. La plus grosse partie du travail est réalisée par quatre personnes du ménage : Lucien, Marie-Françoise, et deux de leurs enfants âgés de 19 et 16 ans. Les trois autres membres du collectif, qui sont des frères et sœurs de Lucien ou de Marie-Françoise remplacent Lucien pour conduire le camion de livraison du pain ou le camion des chevaux ou Marie-Françoise au magasin, les jours de foire ou de concours de chevaux. Lors de ces journées d'été, le travail de boulangerie-pâtisserie et le travail d'élevage équin sont en concurrence dans une période caractérisée par la quantité maximale de travail en boulangerie-pâtisserie du fait de la fréquentation touristique, mais aussi par la disponibilité totale des enfants qui sont en vacances. 
Seules quatre personnes n'appartiennent pas à la famille et sont mobilisées sur des travaux de saison ponctuels : il s'agit du voisin qui aide pour les foins dans le cadre de l'entraide, du maréchal-ferrant pour le parage des chevaux et de deux tondeurs, dans le cadre d'interventions ponctuelles rémunérées.

Le fait que presque toute la famille soit mobilisée ne relève pas seulement de la quantité de travail à faire, c'est aussi une tradition issue de la famille de Lucien, une façon de transmettre des savoir-faire et une identité aux enfants, une façon de vivre en famille. De fait, Lucien et Marie-Françoise créent de l'activité pour donner du travail aux enfants qui le souhaitent. Lucien est fier des savoir-faire de ses fils : "Il fait tout! Les piqûres, je lui ai appris à faire les piqûres. Agneler, il agnèlera une brebis. Il est en stage chez le voisin, il velera une vache, il..., le voisin, il a 85 laitières, il peut s'en aller, lui, il sait traire, [...] Il fera le boulot !... il n'a que 17 ans!». Lucien, qui est allé aux foires avec son propre père, est fier que son fils cadet ait hérité de son talent de négociateur : "Nos fournisseurs pour la pâtisserie, il sait ce qu'il veut, il veut que ce soit tel prix, il veut ça, et ça... ! [...] Il a ça dans le sang".

Travailler ensemble, c'est un plaisir pour Marie-Françoise, qui aime aider le lundi à la bergerie, quand la boulangerie est fermée, elle fait avec les autres, elle ne semble pas indispensable mais elle aime "participer", que " tout le monde participe ". À la remarque sur le fait qu'ils travaillent aussi tous ensemble pour la boulangerie, elle répond : " [La boulangerie] c'est ensemble mais c'est, ce n'est pas pareil. En fait c'est deux choses différentes, lui c'est plus la pratique, la fabrication et moi c'est la vente donc... on ne fait pas la même chose... alors que si je vais soigner on fait la même chose, on travaille, ensemble!"

La répartition des tâches repose sur la polyvalence et la substituabilité des parents et des enfants pour la plupart des tâches relatives à la boulangerie-pâtisserie, à l'élevage équin, à l'élevage ovin. La substituabilité n'est pas totale et elle tient compte des goûts et aptitudes de chacun. Lucien assure le travail quotidien auprès des bêtes quand les enfants ne sont pas là. La répartition des tâches évolue avec l'âge des enfants, qui, même s'ils ne sont pas présents en permanence, participent de plus en plus aux décisions de gestion et d'organisation du travail.

\section{Gestion du temps}

Lucien gère les moutons de manière à y consacrer le moins de temps possible. Il n'hésite pas à reporter des tâches du matin à l'après-midi, d'un jour à l'autre. Le travail avec les brebis est subordonné au travail de la boulangerie. Il essaie de concentrer les tâches reportables sur le week-end et les vacances scolaires, quand les enfants sont là ou au lundi, quand la boulangerie est fermée, ou au jeudi jour sans livraison. Il n'a pas d'ambition en termes de niveau de production.

Aux moments des poulinages et des concours, les travaux avec les chevaux deviennent prioritaires sur tout et toute la famille est mobilisée. La nuit, quand Lucien fabrique le pain, si une jument met bas, il s'interrompt dans la fabrication ou bien il appelle sa femme pour surveiller. 
Lucien apprécie le rythme du travail agricole : "On fait ce qu'on veut! On est à l'air! On est moins pressé qu'à la boulangerie! À la boulangerie, il faut être aux heures précises, tandis que là... si on ne fait pas, on ne le fait pas un jour, on le fait le lendemain, ou le surlendemain... ça va, on est moins pressé!». En parlant des agriculteurs, il met bout à bout les deux expressions "prendre son temps" et "prendre le temps de vivre " : «[...] les agriculteurs, [...] ils ont bien du travail, mais ils prennent leur temps de vivre... Nous on est toujours à fond! ". Lucien déplore l'absence de vacances en famille. Le temps de non-travail est très réduit au profit de l'activité de boulanger-pâtissier, pénible mais très rémunératrice : "C'est vrai, on n'a pas de vie...on n'a pas de weekend! Faut travailler! C'est là que l'on travaille le mieux! Le samedi et le dimanche, les jours fériés, les vacances! Les vacances on ne peut pas partir avec les gamins! C'est vrai on est coincé !". Mais en dehors du temps consacré à la boulangerie, à l'élevage des brebis et des chevaux, Lucien consacre du temps au syndicat de préservation des races de chevaux de trait et à différentes commissions cantonales de chasse.

La dimension familiale de l'engagement dans le travail agricole de Marie-Françoise et Lucien est très forte. Exploitation et pluriactivité ont été héritées. Aujourd'hui, ils travaillent avec leurs enfants et pour que leurs enfants puissent s'installer l'un éleveur, l'autre boulanger. On peut dire que le travail est un mode de vie familial. Le temps libre est réduit mais il existe pour la chasse et le rugby. Le travail agricole n'est pas soumis à des objectifs de rentabilité : l'élevage de brebis sert à installer un fils et l'élevage de chevaux est une passion familiale. Travailler ensemble c'est vivre ensemble.

\section{Discussion-conclusion}

Les exemples analysés ci-dessus montrent que l'engagement de la famille dans le travail agricole prend différentes formes : le cas de Georges illustre une vision très personnelle, où le point de vue et la contribution du conjoint est quasi invisible. Celui de Robert et Béatrice, a contrario montre comment le travail en élevage est " ciment " du couple, tout en autorisant des équilibres de nature différente entre rationalités : plus de poids de la rationalité technique pour l'un, plus de poids pour la rationalité relationnelle pour l'autre, mais qui s'articulent dans le suivi au quotidien du troupeau. Enfin, Lucien et Marie-Françoise illustrent ce que Rault (2005) qualifiait de mode " clanique " de rapport au travail, la combinaison d'activités, et plus particulièrement d'activités agricoles, permettant aux enfants de se réaliser et de - à terme - trouver un emploi au sein d'un système pluriactif géré par une famille élargie. La famille occupe des places diverses dans le collectif de travail (de minoritaire à majoritaire) et limite le temps dédié à l'élevage ou non, notamment dans le cas où le temps pour l'élevage est perçu comme du temps en famille. La dimension familiale est donc toujours présente comme le montrent aussi Terrier et al. (2012) en dépit du fait que la famille non coexploitante régresse sans cesse (Giroux, 2011).

Ces formes d'engagement sont très visibles chez les pluriactifs, notamment du fait de la parfois moindre importance de la rationalité économique dans le rapport à l'élevage et dans la façon de conduire l'élevage et d'organiser le travail, de gérer le 
temps. Ces formes d'engagement se matérialisent notamment dans la façon de gérer les temps de travail agricole, non agricole, en famille. Ce qui fait partie du temps en famille ou du temps de travail en élevage ou du temps de non travail differe selon les personnes : pour certains, le temps de l'élevage c'est du temps en famille, en couple, avec les enfants, c'est du temps libre, car les horaires sont libres, car ils ont choisi. Toutefois, ces formes d'engagement ne sont a priori pas propres aux pluriactifs. On les retrouve dans des études qui portent sur des agriculteurs dits "à temps plein »: pour ce qui est de la projection maternelle sur les relations entretenues avec les bêtes (Salmona, 1994), de la transmission de l'état de paysan (Barthez, 1996), du travail agricole comme façon de vivre ensemble en famille (Macombe, 2007). Toutefois, la forme d'engagement relevant de la réalisation personnelle sans pression économique, illustrée par le cas de Georges relève sans doute davantage des pluriactifs qui ont choisi de faire une activité agricole et qui n'en attendent pas de revenu pour la famille. Les situations d'agriculteurs seuls permanents sur la ferme, à faible contribution aux revenus du ménage à certaines périodes de la trajectoire de l'exploitation (par exemple pendant les emprunts pour l'installation) ne sont pas exceptionnelles (Bégon et al., 2009), tout comme la nécessité de combiner activité agricole, activité du conjoint et prise de congés, dans les situations où le conjoint véhicule les normes du travail salarié (Dufour et Dedieu, 2010).

Ces exemples invitent à une posture compréhensive pour caractériser les attentes des agriculteurs vis-à-vis de leur travail, et de leur temps de travail, et aborder la question de l'articulation entre la famille et l'exploitation dans les actions de recherche et de conseil visant à améliorer les conditions de vie au travail des éleveurs (Fiorelli et al., 2012). Ils sont susceptibles d'aider les conseillers à entendre, aborder, écouter et prendre en compte des dimensions du travail qu'ils qualifient d'intimes ou de personnelles et qu'ils jugent difficiles à aborder (Kling-Eveillard et al., 2012) pour construire avec l'éleveur son problème de travail et l'accompagner dans sa résolution (Mallot et Voisin, 2006). En cela, notre démarche et nos résultats proposent des grilles d'analyse très complémentaires des travaux de recherche et développement qui expriment les enjeux d'amélioration des conditions de travail en élevage, qualifiée de vivabilité, en termes de durée et de productivité du travail (Cournut et Chauvat, 2012). Plus largement, ce qui est en débat, c'est un double défi pour analyser les systèmes agricoles : (i) celui de considérer l'éleveur non seulement comme un pilote technico-économique mais aussi comme un organisateur du travail et un travailleur sensible qui se réalise dans son travail (Cerf et Sagory, 2004 ; Dedieu et Servière, 2012), mais aussi (ii) de considérer derrière la figure du chef d'exploitation, les différents membres du groupe familial, ce que chacun porte, y compris de différent comme raison de travailler en élevage. En effet, le fait que le travail d'élevage soit intimement lié à la vie familiale peut être vécu par les acteurs de façon très différente au fil du temps (Terrier, 2013). L'imbrication entre travail, famille et vie privée peut rendre difficiles, voire impossibles sans rupture, les désirs d'autonomie, d'innovations ou de changements. Cette question temporelle pourrait sans doute être prise en compte en repensant la place de l'élevage familial au sein de l'élevage paysan. L'élevage paysan, celui qui innove et est en prise avec son siècle, doit-il être encore fondé sur la famille, ou celle-ci n'est-elle qu'une option parmi d'autres ? 


\section{Bibliographie}

Barthez, A., 1996. Les relations de l'agriculteur avec son travail : une longue histoire, de forts changements actuels, Travaux et innovations, 25, 15-17.

Bégon, M., Pailleux, J.-Y., Joly, N., Lémery, B., Dedieu, B., 2009. Les chemins pour durer en élevage bovin laitier : diversité des logiques d'action sur le long terme en Ségala (Massif Central), in, $16^{e}$ Rencontres Recherches Ruminants, décembre 2010, Paris. Consultable sur le site : http://www.journees3r.fr/ IMG/pdf/2009_02_05_Begon.pdf

Bessière, C., 2008. "Se marier pour aller jusqu'au bout ensemble? » ruptures conjugales et transmission des exploitations agricoles dans la lignée, Revue d'Études en Agriculture et Environnement, 88, 3, 47-70.

Blanchemanche, S., 2000. La combinaison d'activités professionnelles des ménages agricoles. L'exemple du département de l'Isère, Thèse de doctorat, Université de Paris X, Nanterre.

Cerf, M., Sagory, P., 2004. L'agriculture, un secteur critique dans les sociétés, in Falzon, P. (dir.) Ergonomie, Paris, PUF, 491-499.

Cournut, S., Chauvat, S., 2012. L’organisation du travail en exploitation d'élevage : analyse de 630 Bilans Travail réalisés dans huit filières animales, Inra Productions Animales, 25, 2, 101-112.

Couzy, C., Dockès, A.-C., 2006. Multiplicité des métiers, diversité des modèles de référence : un éclairage sur les transformations des métiers des agriculteurs, in, $13^{\text {es }}$ Rencontres Recherches Ruminants, décembre 2007, Paris. Consultable sur le site : http://ieparis5.inst-elevage.asso.fr/html28/IMG/pdf/2006_3_metier_ travail_01_Couzy.pdf

Dedieu, B., Servière, G., 2012. Vingt ans de recherche - développement sur le travail en élevage : acquis et perspectives, Inra Productions Animales, 25, 2, 85-100.

Dejours, C., 1998. Travailler n'est pas déroger, Travailler, 1, 5-12.

Dejours, C., 2009. Travail vivant, T2 : Travail et émancipation, Paris, Payot.

Dufour, A., Dedieu, B., 2010. Rapport au temps de travail et modes d'organisation en élevage laitier, Cahiers Agricultures, 19, 5, 377-382.

Dufour, A., Courdin, V., Dedieu, B., 2010. Femmes et travail en couple : pratiques et représentations en élevage laitier en Uruguay et en France, Cahiers Agricultures, 19, 5, 371-376.

Fillonneau, G., 2012. Du déclassement professionnel à la désaffection pour le métier. Le cas des producteurs laitiers du Marais Poitevin, Revue d'Études en Agriculture et Environnement, 93, 3.

Fiorelli, C., 2010. L'aménagement des conditions de vie au travail des éleveurs : proposition d'un cadre d'analyse des relations entre rapport subjectif et organisation $d u$ travail en élevage. Étude de cas chez des éleveurs pluriactifs, Thèse de doctorat, Paris, AgroParisTech. 
Fiorelli, C., Dedieu, B., Pailleux, J.-Y., 2007. Explaining diversity of livestock-farming management strategies of multiple-job holders : importance of level of production objectives and role of farming in the household, Animal, 1, 08, 1209-1218.

Fiorelli, C., Mouret, S., Porcher, J., 2012. Les rationalités du travail avec les animaux d'élevage : produire, vivre ensemble et se construire. Inra Productions Animales, 25, 2, 181-192.

Fiorelli, C., Porcher, J., Dedieu, B., 2010. Un cadre d'analyse des compromis adoptés par les éleveurs pour organiser leur travail, Cahiers Agricultures, 19, 5, 383-390.

Giroux, G., 2011. Premières tendances recensement agricole 2010, Agreste Primeur, 266,

Hostiou, N., Dedieu, B., 2012. A method for assessing work productivity and flexibility in livestock farms. Animal, 6, 5, 852-862.

Kling-Eveillard, F., Cerf, M., Chauvat, S., Sabatté, N., 2012. Le travail, sujet intime et multifacette : premières recommandations pour l'aborder dans le conseil en élevage, Inra Productions Animales, 25, 2, 211-220.

Laignel, G., Fiorelli, C., Dedieu, B., Porcher, J., 2008. Éleveurs pluriactifs ovins viandes: Analyse technico-économique et lien aux rationalités du travail, in, $15^{\text {es }}$ Rencontres Recherches Ruminants, Paris. Consultable sur le site : http:// www.journees3r.fr/IMG/pdf/2008_08_economie_11_Laignel.pdf

Lémery, B., 2005. Les agriculteurs dans la fabrique d'une nouvelle agriculture, Sociologie du travail, 45, 1, 9-25.

Macombe, C., 2007. Work : A necessary sacrifice or a suffered chore ? Labor and farm continuity in alternative agriculture in France, Renewable Agriculture and Food Systems, 22, 4, 282-289.

Madelrieux, S., Dedieu, B., 2008. Qualification and assessment of work organisation in livestock farms, Animal, 2, 3, 435-446.

Madelrieux, S., Dedieu, B., Dobremez, L., Girard, N., 2009. Patterns of work organisation in livestock farms : the ATELAGE approach, Livestock science, 121, $1,28-37$.

Mallot, M., Voisin, J., 2006. Conseil travail en élevage ovin : bilan de ces expériences et évolution du métier de technicien, in Sommet de l'Élevage, octobre 2006, Clermont-Ferrand.

Mouret, S., 2012. Élever et tuer des animaux, Paris, France, PUF.

Porcher, J., 2002. Eleveurs et animaux : réinventer le lien, Paris, PUF.

Rault, G., 2005. Approche sociologique du travail en élevage et questions sur le conseil agricole, in Actes du séminaire AFPF - Elevage, prairies, travail, Paris, 20 octobre 2005, 29-35.

Rémy, J., 2011. De la célébration de l'agriculture familiale à la promotion de l'agriculteur, Pour, 212, 165-178.

Salmona, M., 1994. Les paysans français : le travail, les métiers, la transmission des savoirs, Paris, L'Harmattan. 
Sens, S., Soriano, V., 2001. Parlez-moi d'élevage. Analyse de représentations d'éleveurs, Dijon, Educagri Éditions.

Terrier, M., 2013. Réalités de l'exploitation familiale au prisme du temps long. proposition d'un cadre d'analyse interdisciplinaire et illustrations en exploitations d'élevage bovin lait dans le Vercors, Paris, AgroParisTech.

Terrier, M., Madelrieux, S., Dufour, A., Dedieu, B., 2012. Saisir la diversité des formes d'articulation entre la famille et l'exploitation agricole : une grille de lecture, Revue d'Études en Agriculture et Environnement, 93, 3, 299-322. 